\title{
18F-FDG-PET and PET/CT as a diagnostic method for Ewing sarcoma: a systematic review and meta-analysis.
}

\author{
Ishith Seth ${ }^{1}$, Nimish Seth ${ }^{2}$, Gabriella Bulloch ${ }^{3}$, Adrian Siu ${ }^{1}$, Allen Guo ${ }^{4}$, Rukhmini \\ Chatterjee $^{1}$, Michael MacManus ${ }^{5}$, and Leo Donnan ${ }^{6}$ \\ ${ }^{1}$ Illawarra Shoalhaven Local Health District \\ ${ }^{2}$ Alfred Health \\ ${ }^{3}$ The University of Melbourne Melbourne Medical School \\ ${ }^{4}$ University of New South Wales Faculty of Medicine \\ ${ }^{5}$ Peter MacCallum Cancer Centre \\ ${ }^{6}$ The Royal Children's Hospital Melbourne
}

August 7, 2021

\begin{abstract}
Purpose: The aim of this study was to evaluate the diagnostic accuracy of 18-fluorodeoxyglucose-positron emission tomography (18F-FDG-PET) and positron emission tomography/computed tomography (PET/CT) in imaging primary and metastatic lesions in Ewing sarcoma (ES). Methods: PubMed, Cochrane, Scopus, and Web of Science were searched for relevant studies. Data concerning 18F-FDG-PET/CT diagnostic accuracy were extracted and then analysed using Open Meta-analyst software. Reported diagnostic accuracy outcomes included sensitivity, specificity, negative likelihood ratio (NLR), positive likelihood ratio (PLR), and diagnostic odds ratio. Results: 31 studies with a total of 735 patients were included in this meta-analysis. The sensitivity and specificity of 18F-FDG PET/CT were: $92.6 \%$ and $74.1 \%$ for total ES lesions, $96.7 \%$ and $68.3 \%$ for ES primary lesions, $76.1 \%$ and $92.4 \%$ for lung metastasis, $83.9 \%$ and $93.2 \%$ for bone metastasis and $89.9 \%$ and $92.6 \%$ for ES recurrence respectively. Conclusion: 18F-FDG PET/CT is sensitive and accurate in diagnosing, staging, and detecting the recurrence of ES compared to non-PET imaging. It has high specificity for diagnosing recurrence of ES as well as lung and bone metastases.
\end{abstract}

\section{${ }^{18}$ F-FDG-PET and PET/CT as a diagnostic method for Ewing sarcoma: a systematic review} and meta-analysis.

Authors: Ishith Seth, $\mathrm{MD}^{1^{*}}$, Nimish Seth, $\mathrm{MD}^{2}$, Gabriella Bulloch, BMSc${ }^{3}$, Adrian Hang Yue Siu, MD ${ }^{1}$, Allen Guo ${ }^{4}$, Rukmini Chatterjee, MD ${ }^{1}$, Michael MacManus, FRANZCR ${ }^{5}$, Leo Donnan, FRACS ${ }^{6}$

${ }^{1}$ Illawarra Shoalhaven Local Health District, Wollongong Hospital, Wollongong, New South Wales, 2500, Australia.

${ }^{2}$ Department of Orthopaedic Surgery, The Alfred Hospital, Melbourne, Victoria, 3004, Australia.

${ }^{3}$ Faculty of Science, Medicine and Health, University of Melbourne, Victoria, 3010, Australia.

${ }^{4}$ Faculty of Science, Medicine and Health, University of New South Wales, New South Wales, 2052, Australia.

${ }^{5}$ Department of Radiation Oncology, Peter MacCallum Cancer Centre, Melbourne, Victoria, 3000.

${ }^{6}$ Department of Orthopaedic Surgery, The Royal Children's Hospital, Melbourne, Victoria, 3052, Australia.

*Corresponding to: 
Ishith Seth, MD, Division of Surgery, Illawarra Shoalhaven Local Health District, Wollongong, New South Wales, 2500 Australia, Tel.: +61434259479, Email: ishithseth1@gmail.com

Main text word count : 3764 words

Abstract word count : 215 words

Brief running title: Accuracy of FDG PET/CT in Ewing Sarcoma

Keywords: 18 F-FDG PET; PET/CT; diagnostic accuracy, Ewing sarcoma; meta-analysis; metastasis; recurrence.

Tables : 2

Figures : 6

Supplementary file : 1

Abbreviations Table

\begin{tabular}{|c|c|}
\hline Abbreviation & Definition \\
\hline${ }^{18} \mathrm{~F}$ & 18 Fluorine \\
\hline${ }^{18} \mathrm{~F}-\mathrm{FDG}$ & 18 fluorine-fluorodeoxyglucose \\
\hline $\mathrm{BMB}$ & bone marrow biopsy \\
\hline CI & confidence interval \\
\hline $\mathrm{CT}$ & computed tomography \\
\hline DOR & diagnostic odds ratio \\
\hline ES & Ewing Sarcoma \\
\hline FDG & fluorodeoxyglucose \\
\hline FP & false positive \\
\hline HL & Hodgkin Lymphoma \\
\hline$I^{2}$ & I square \\
\hline IV & intravenous \\
\hline $\mathrm{MBq}$ & mega Bequerel \\
\hline $\mathrm{mCi}$ & milli Curie \\
\hline MRI & magnetic resonance imaging \\
\hline NHL & Non-Hodgkin Lymphoma \\
\hline NLR & negative likelihood ratio \\
\hline NPV & negative predictive value \\
\hline PET & positron emission tomography \\
\hline PLR & positive likelihood ratio \\
\hline PPV & positive predictive value \\
\hline PRISMA & Preferred Reporting Items for Systematic Reviews and Meta-Analyses \\
\hline QUADAS & quality assessment of diagnostic accuracy studies \\
\hline $\mathrm{ROC}$ & receiver operating characteristic \\
\hline SEMI & semiquantitative \\
\hline SPECT & single-photon emission computed tomography \\
\hline SROC & summary receiver operating characteristic \\
\hline $\mathrm{TN}$ & true negative \\
\hline $\mathrm{TP}$ & true positive \\
\hline${ }^{18}$ F-FDG PET & 18 fluorine-fluorodeoxyglucose positron emission tomography \\
\hline${ }^{18} \mathrm{~F}-\mathrm{FDG} \mathrm{PET} / \mathrm{CT}$ & 18 fluorine-fluorodeoxyglucose positron emission tomography / computed tomography \\
\hline 99mTc-MDP & Technetium 99m-methyl diphosphonate \\
\hline $\mathrm{PET} / \mathrm{CT}$ & positron emission tomography/ computed tomography \\
\hline PRISMA-DTA & Preferred Reporting Items for Systematic Reviews and Meta-Analyses of Diagnostic Test Accuracy \\
\hline
\end{tabular}




\section{Hosted file}

Ewing sarcoma manuscript.docx available at https://authorea.com/users/429455/articles/53315518f-fdg-pet-and-pet-ct-as-a-diagnostic-method-for-ewing-sarcoma-a-systematic-reviewand-meta-analysis 